\title{
Proceeding
}

Supplementary Issue: Summer Conferences of Sports Science. Costa Blanca Sports Science Events, 25-26 September 2020. Alicante, Spain.

\section{Tactical learning control system in the Honduras handball team}

\author{
REIDEL CORDOVÉS-PEINADO ${ }^{1} \unlhd$, FAUSTO RICARDO BAIDE-ORDOÑEZ¹, MARISNEL LAO-CORDERO² \\ ${ }^{1}$ National Autonomous University of Honduras, Tegucigalpa, Honduras \\ 2University of Oriente, Santiago de Cuba, Cuba
}

\begin{abstract}
From a scientific perspective, this research addresses a current and relevant topic for sports training in handball. Part of the determination of the deficiencies of the evaluation in the tactical activity in the team and recognizes its multifactorial nature. Consequently, it provides a control system supported by tests, tests and records in correspondence with the defined indicators that characterize the tactical learning process in the handball team, evaluating all its actors, which allows defining quality in the process. Its conception contributes to the development of the tactical learning process is essential as an object of study, by offering the methodological didactic tools in function of transforming the modes of action in the coaches and raising the quality of the training of the handball team. The relevance and feasibility are corroborated from the application with the intentionally chosen sample, thus being the Honduran handball team classified for the Central American and Caribbean games.
\end{abstract}

Keywords: Tactics; Learning; Control system; Multifactorial evaluation.

\section{Cite this article as:}

Cordovés-Peinado, R., Baide-Ordoñez, F.R., \& LaO-Cordero, M. (2020). Tactical learning control system in the Honduras handball team. Journal of Human Sport and Exercise, 15(4proc), S1500-S1511. doi:https://doi.org/10.14198/jhse.2020.15.Proc4.46

Corresponding author. National Autonomous University of Honduras, 11101 Tegucigalpa, Honduras. https://orcid.org/0000$\underline{0002-0894-7536}$

E-mail: reidel.cordoves@unah.edu.hn

Abstract submitted to: Spring Conferences of Sports Science. Costa Blanca Sports Science Events, 19-20 June 2020. Alicante, Spain.

JOURNAL OF HUMAN SPORT \& EXERCISE ISSN 1988-5202

(c) Faculty of Education. University of Alicante

doi:10.14198/jhse.2020.15.Proc4.46 


\section{INTRODUCTION}

The research deals with the sport of handball, which is characterized by invasion, opposition, collaboration and cohesion, in order to score the goal in opposing fields. In the dynamic exchange in the training and competition scenarios, the intention to break the opponent's tactic prevails. In this sense, unity and communication are determining factors in solving the various situations to which its practitioners are exposed in attack or defence conditions. Hence the need for an integrative perspective in the preparation of team players that involves physical, technical, tactical, psychological and theoretical components.

This sporting activity has acquired great relevance in the world sports movement, taking into account its evolution from what constituted a rough game with supremacy in the use of force to the detriment of technique and tactics. However, the evolutionary transition to the present shows the prospect of a harmonious and creative tactical game. In this context, the constant variations of the offensive and defensive game systems are weighed very quickly in the effort to achieve victory. Such practice demands impose the constant improvement of the handball teaching-learning process (PEA) and in a particular way call for a quality evaluation.

However, their results do not particularize in handball and reveal the need to delve into tactics and other aspects of evaluation in this sport. On the other hand, they assume evaluation as part of control, a theoretical position not currently shared in the pedagogical context, and they contradict the theoretical position of the present study.

The theoretical assumptions that have dealt with important issues regarding evaluation in the sport of handball, and some in the context of tactics, are avant-garde such as: Refoyo (2001), Feu (2006), Mesa (2007). Román (2008), Antón (2010), Quintana. (2014). In essence, they provide valuable input about evaluation and recognize the need to direct it toward tactics. However, their approaches connote performance in training or competitive outcome, while learning is left out of evaluation in both scenarios.

The most recent studies on the subject systematize their results from the function of control in the completion of actions, being a consistent trend in many cases of the aforementioned studies, but providing statistical and computer technology packages to support their studies, to mention some, Casanova, Olavarrieta, Núñez (2017) stand out. González (2015). LLC, M. (2017). Lo Giudice, (2016). Salas, Mendo, (2016).

Therefore, measurement prevails through exercises in training and their actions in the game. From this perspective, the limited representativeness of this result in the teaching-learning process (PEA) is ignored, since a positive result in the competition does not always show its quality.

On the other hand, there are limitations in the analysis of compliance with the planning system, based on the establishment of quantitative goals through volumes of work. Under such conditions, the objectives set for the tactical preparation stages are not subject to a thorough quality assessment.

Added to the above is the limited use of the comprehensive nature of the evaluation. In particular, the diagnostic and developmental functions, in addition to the instructive and educational functions, are not envisioned in their entirety. Such practice contradicts the maxim of the PEA of tactical activity by demonstrating the need not to ignore these aspects, since observing them results in quality. 
Thus, in this study it presumes shortcomings such as: limited deepening of the evaluation, with emphasis on performance and results in training and competition scenarios, prevalence of quantitative control, to the detriment of other evaluation functions in the PEA. In order to propose, from the proposal of a control system, the analysis of the effectiveness of tactical learning in handball teams.

\section{METHOD}

A pre-experiment is carried out for the evaluation of learning in the tactical activity in the handball team. In this case, on the basis of descriptive statistics in order to analyse the quantitative results of the instruments applied to the sample, tabulation and data processing; through the absolute, relative, cumulative and percentage frequencies.

\section{Participants}

Two groups are proposed for the Honduran men's handball preselection, which was prepared for the Central American and Caribbean games in 2018, in this sense, there are a total of 26 players, which will be divided into groups $A$ and $B$, being control and study respectively.

\section{Control system procedure for the analysis of the evaluation of tactical learning}

Its purpose is to insert it into the reference system from its objectivity and to contribute to satisfying the didactic deficiencies of pedagogical practice. Shows a high degree of adaptability to the expectations and demands of professional performance and cognitive development of the team. For its projection it links the physical means, the ways of interaction between the tests and the characteristics of the link of the concrete tactical situation in which the learning will be manifested (training-game).

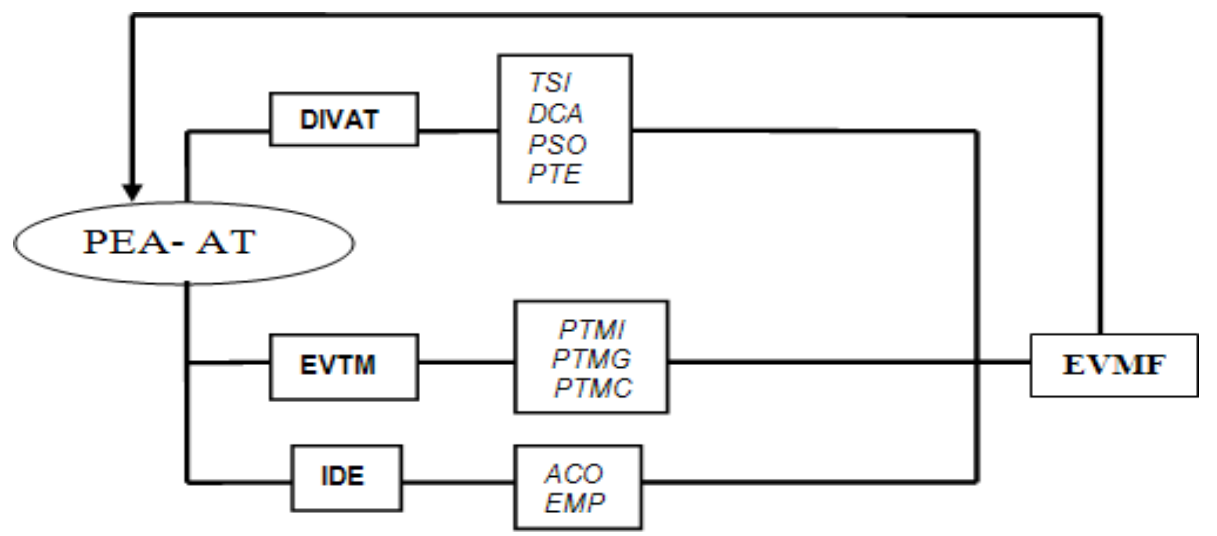

Legend: PEA - AT - Teaching-learning process in the tactical activity. DIVAT - Comprehensive study related to tactical learning in the handball team. PSI Psychological tests. DCA - Determination of learning ability. PSO - Sociological characterization. PTE Theoretical test EVTM - Motor tactical evaluation. PTMI - Individual motor tactical performance tests. PTMG - Group motor tactical performance test. PTMC - Team Motor Tactical Performance Test. MDE - Didactic mediation of the coach. ACO - Analysis of the fulfilment of the objectives. AMP - Analysis of methods and procedures. EVMF - Multifactorial Assessment

Figure 1. The control system for learning in tactical activity in the handball team.

DIVAT - Name the group of tests corresponding to the first subsystem. For this, it includes the instruments and indicators to offer the coach the initial state of the favourable conditions or not, to achieve learning in tactical activity. 
In the DIVAT the evaluative nature of the tests varies. Those of a psychological type, for example, have to express quantitative results that require qualitative interpretation to be compatible with the rest of the tests. The evaluation of the subsystem will be determined by the results of the total tests evaluated. The parameters according to the data that are reflected are categorized as favourable (when the results in more than three tests are considered good); unfavourable (when the result in two tests is good) and unfavourable (in one or none).

TSI - Refers to psychological tests in relation to tactical activity. It does not constitute a static tool, that is to say, the pedagogical group chooses the ones that it will apply according to the interests and needs.

DCA - It is based on an observation record from which the learning capacity is determined from the indicators of consistency and progression.

PSO - Offers a sociological type test which allows determining the group dynamics of the team and evaluating the group conditions given in the team. Indicators of: cohesion, cooperation, interaction, structure and goals are proposed. It is important to note that the test will be based on observation through exercises with the participation of two or more players, as well as on the games selected for measurement.

PTE - Groups the tests aimed at checking the players' theoretical tactical knowledge to solve the problems of this type during training and play. They are done in writing and allow to determine the cognitive range they present. In the oral variant they try to mediate with practical interaction in the concrete learning conditions; it is recommended to apply them in a state of exhaustion to force the player to think about these conditions. Similarly, its implementation is considered possible in intermediate breaks between unit activities, whether physical, technical or tactical.

EVTM - Names the group of tests for the evaluation of motor tactical performance, supported by measurement records based on observation. They allow to control, the indicators relative to the manifestations of the learning of the tactical activity in handball. Through which the spaces for incidence and the ways or resources to achieve their results are evidenced. The tactical demands that allow considering the effectiveness of the actions that are the object of learning start from the whole procedure and not from the end as expressed by the nature of the performance.

PTMI - Represents the individual tactical performance test, the results of which allow to determine the significance of learning in the player.

In the same way, the observation of how the goal is reached is promoted, in which the effective action in which the player runs by himself, a group or the team is taken into account, an action in which the player is in possession of the ball, and makes a 1vs1 attack, and through the feint he manages to evade the mark, to reach the door, individual tactical action is effective.

PTMG - It proposes the group tactical performance test, it is determined by the conformation of the groups both in number of players and in the particularities of the relationships, according to the proximity between the players in the training or game scenarios. The result in this test can give prognostic advances in team control, which if positive will express agreement with cooperative learning. It is inferred in the degree of participation of the players, to reach the goal or avoid the goal, which in turn guarantees the effectiveness of the action, to happen that the player carrying the mobile arrives at the opposite goal, it is important to highlight the participation of the companions that almost certainly, allowed this success. In this sense, from the 
offensive, it is exemplified through the actions of splits that promote the creation and occupation of space for the final penetration towards the goal area.

In the defensive space, the obstruction is achieved by the collaboration of the neighbouring players, which allow anticipating the offensive action, reaching its objective.

PTMC - Refers to the collective tactical performance test related to the actions where the generality of the team intervenes, from the offensive and defensive game systems that allow determining the developing essence of learning.

To determine learning, it is proposed from the determination of its effectiveness by the following:

$$
\begin{gathered}
\text { Learning effectiveness }=(R A * 100) / R A \\
\text { Creativity Level }=(R C * 100) / 0
\end{gathered}
$$

Represent: RA: Right answers. O: Number of Opportunities. RC: Creation Responses.

The effectiveness of learning will be determined based on the following evaluative criteria:

Table 1. Effectiveness of learning for field players.

\begin{tabular}{llll}
\hline Learning effectiveness & \multicolumn{3}{l}{ Creativity Level } \\
\hline Very good & $+85 \%$ & Very good & $+60 \%$ \\
Good & $69-84 \%$ & Good & $59-40 \%$ \\
Regular & $51-68 \%$ & Regular & $39-20 \%$ \\
Evil & $-50 \%$ & Evil & $-19 \%$ \\
\hline
\end{tabular}

Table 2. Effectiveness of learning for goalkeepers.

\begin{tabular}{llll}
\hline Learning effectiveness & & Creativity Level & \\
\hline Very good & $+50 \%$ & Very good & $+35 \%$ \\
good & $30-49 \%$ & Good & $20-34 \%$ \\
Regular & $15-29 \%$ & Regular & $9-19 \%$ \\
Evil & $-15 \%$ & Evil & $-8 \%$ \\
\hline
\end{tabular}

MDE - Term related to the didactic mediation of the trainer, obtained as the essence of the analysis of the results of the process. It proposes an observation protocol and assumes as indicators the didactic components that intervene and establishes its correspondence with the quality of learning, based on the relationship with the other components.

ACO - Expresses the analysis of the fulfilment of the objectives, based on the assessment of the relationship between the intended purpose and team learning. It favours the determination of the correspondence between the learning foreseen and those not foreseen in the PEA of the tactical activity in the handball team.

AMP - Reflects the analysis of the methods and procedures required to favour the conception and correct direction of the PEA from the mediating role of the coach. 
EVMF - Represents the results of the multifactorial evaluation, at the same time it assumes the principle of the integration of different means for its implementation. The evaluative resources to be used are determined from the objectives to be controlled. It starts from the recognition of the complexity of the PEA of the tactical activity, to assume the diversity of the aspects to be evaluated. This criterion agrees with the assessment of the media, considering its limited reliability based on the results to check the quality of learning. Hence the need for its systemic integration, based on a selection in close correspondence with the particular reality of each coach.

Through the control system, the data obtained in the tests and media tests are linked, the particularities of which will depend on the correlation of results from each component to the generality of the system. In this way, the factors influencing learning in tactical activity are integrated into a single evaluative criterion.

The methods to support the group of tests of the proposed control system must express their coherence with those assumed by the PEA. In this sense, it is necessary to be consistent with the developer vision assumed by the evaluation. Accordingly, they are declared below.

Observation: it is proposed as a process verification tool, focused on the description of what happened in the training or game scenarios. Its use is based on the assessment and verification procedures.

Measurement: it is projected as a tool for the precise registration of data. Part of the assignment of values to the indicators established by the observation, through the combination of the data in relation to the approaches established at the time of control. At the same time, it constitutes a quantitative value assessment aspect. Therefore, it requires to be subjected to a qualitative interpretation, in correspondence with the nature of the subsystems of the model.

\section{RESULTS}

In general, in the test of 341 possible solutions (162 offensive and 179 defensive), 217 correct ones are reached, representing $63.63 \%$. This data indicates an average level of attention volume and the level of efficiency in the ability to perceive the greatest number of simultaneous situations.

Decision-making: from their results the level of security of the players is inferred, which placed the team at level II. In particular, the front-line players averaged for $77.58 \%$, the second line for $64.46 \%$ and the goalkeepers for $69.67 \%$. The fluctuation manifests itself in $70.57 \%$ and expresses the resulting discomfort, according to the test scale.

In the case of learning capacity, the results of the causal diagnosis of the investigation, relative to the equipment object of the pre-experiment, are considered pertinent.

The qualitative correlation of the results obtained in the tests allows to infer the location of the conditions for learning in tactical activity in the handball team, in the unfavourable range, according to the objectives. Such assessment is based on the concentration of three of the four components of the subsystem typified in it. Based on the DIVAT considerations, the PEA of the tactical activity is conducted.

The formative evaluation is broken down into two moments. These offer various patterns of results from the coach's didactic mediation. In the intermediate, individual and group tactical performance is valued; while the final one focuses on the collective. In this way, the evolution of the objective is evaluated and its fulfilment is 
analysed. The tests are applied in the special preparation stage, seventh basic developer mesocycle with a total of six microcycles of 36 units, in intervals of 18 training sessions in the morning hours.

Table 3. Tactical learning evaluation record.

\begin{tabular}{|c|c|c|c|c|c|c|c|c|c|c|}
\hline \multirow[t]{2}{*}{ Player } & \multicolumn{3}{|l|}{ Shape } & \multicolumn{4}{|c|}{$\begin{array}{l}\text { Participants in the } \\
\text { Knowledge appropriation }\end{array}$} & \multicolumn{3}{|c|}{$\begin{array}{l}\text { Level at which } \\
\text { Manifest learning }\end{array}$} \\
\hline & ERCA & SJ & $\mathrm{J}$ & 1 & IP & IC & IPC & $E$ & $\mathrm{RC}$ & $A C$ \\
\hline 1 & & $x$ & & & $x$ & & & $x$ & & \\
\hline 2 & & $x$ & & & & $x$ & & $x$ & & \\
\hline 3 & & $x$ & & & & & $x$ & $x$ & & \\
\hline 4 & & $x$ & & & & $x$ & & & $x$ & \\
\hline 5 & & $x$ & & $x$ & & & & & & $x$ \\
\hline 6 & & $x$ & & $x$ & & & & & & $x$ \\
\hline 7 & & $x$ & & $x$ & & & & & & $x$ \\
\hline 8 & & $x$ & & & & & $x$ & $x$ & & \\
\hline 9 & & $x$ & & & & $x$ & & & $x$ & \\
\hline 10 & & $x$ & & & & $x$ & & & $x$ & \\
\hline 11 & & $x$ & & & $x$ & & & & & \\
\hline 12 & & $x$ & & & & $x$ & & & $x$ & \\
\hline 13 & & $x$ & & & & $x$ & & $x$ & & \\
\hline 14 & & $x$ & & & $x$ & & & $x$ & & \\
\hline
\end{tabular}

Legend: ERCA. - Isolated character repetition exercises. SJ - Game situations. J. - Game I.- Independence. IP. - Teacher intervention. IC. Partner Intervention. CPI. Teacher and classmate intervention. E. - Execute RC. - Reacts to the imposed situation, on the contrary. AC. - He anticipates and creates situations. PPG - Participates a portion of the group GT- Group in its entirety CG. - Collaboration of another group CGP - Collaboration of another group and the teacher. ET - Team in its entirety CE - Collaboration of another team CEP - Collaboration of another teaching team.

In the first application of the EVTM, the observation of the selected exercises and three preparation games were conceived, with the subsequent analysis of the coach's didactic mediation. From the individual offensive point of view, the players averaged a regular evaluation, for a relative effectiveness of $67.69 \%$. The creation levels behaved $17.88 \%$ of hits in the actions in relation to the number of implicit opportunities. In general, a total of 153 actions were carried out among the repetitions carried out by each member of the team, of the 226 elaborated by the coach with the possibility of manifesting themselves in game situations, for a $59.33 \%$ effectiveness. Showing fluctuation between the evaluation result between training and game situations.

On the defensive, the values averaged $47.76 \%$, which is why it is considered Bad, according to the times of intervention of the player before the attack with the determined action and the number of opportunities consisting of a total of 344 actions. They show the difficulties from a defensive point of view. It is worth noting the role played in the defensive zones by the front row players.

The results in the evaluation of the goalkeepers averaged a regular evaluation, oscillating by a $25.47 \%$ effectiveness. They showed difficulty in taking advantage of offensive opportunities, essentially in the counterattack pass.

The aforementioned, reveals a balance between offense and defence, by not showing notable differences. However, it is recommended to pay attention to the specific aspects that do not satisfy the average. Such is the case of unchecking without the ball on offense, which can directly affect the occupation of space at the croup level. For its part, the defence interception requires follow-up as it shows limited anticipation on the 
part of the players. Response creation patterns fall short of favourable, in contrast to meaningful learning. In essence, they manifest a dedication of minimal work time to tactics and low levels of creation.

Group tactical performance is assessed by observing four groups, according to the boundaries of the players in space. Two are defined in each line of attack. On offense, the occupation of space prevails, which shows the ability to uncheck between players with a $68.36 \%$ effectiveness in the opportunities-times ratio, evaluating these regular results. Creation levels fluctuated by $28.35 \%$. Lacks in cohesion between players are declared, through the few incidents in the linking actions.

The defensive aspect shows a low level of relationships between the players. They denote the difficulties in the change of opponent, the help and the doubling. This accounts for the lack of cohesion, and consequently, reveals communication impairments. Deterrence manifests worrying values, with only two effective interventions out of eight possible.

The observation described shows that instability in the incidence of the subjects in the actions constitutes a regularity. In this sense, two are located in the execution learning level and the rest, in equal numbers, located in the reaction and creation level. The foregoing accounts for the difficulties in group motor tactical learning.

In match conditions, observation of tactical learning was carried out with two teams $A$ and $B$. The learning foreseen in A was evaluated as Bad, taking into account $44.77 \%$ effectiveness, although it shows the prevalence in the counterattack over the positional game. The aforementioned shows the players' ability to transition, but positional play is limited. This in turn reveals a lack of cohesion between players. From the defensive point of view, a low level of relations between the players is observed, as well as difficulties in retreating. This shows the correspondence with the positive results of team $A$ in the counterattack.

However, team B showed more encouraging results, as there is evidence of a better mastery of the defensive systems put into practice during matches. In this sense, they reflect improvement in group aspects such as deterrence, changes of opponents and collaboration with the effectiveness of defensive systems, their effectiveness was around $53.33 \%$ considered fair.

From the difficulties in the evaluated tactical indicators, the process is adjusted, in order to improve the learning results. Therefore, two series of observations are incorporated into the diagnosis, which will show the importance of learning in the players, after the analysis of the coach's didactic intervention. Seven training sessions related to tactical preparation were visualized in all its manifestations. The results obtained in each indicator, from the determination of the absolute frequencies and percentages.

The interpretation of the quantitative data related to the intermediate application of the EVTM, allows inferring the shortcomings in the methodological treatment of the preparation of the coaches' tactics and their direct responsibility in the results of the first evaluation series. In this sense, a regression is observed from the adequate to the unsuitable. The adjustment in the direction of the PEA and the transformation of the modes of action is imposed, in order to influence the correspondence between the control system and its way of conceiving it. In the latter, the former exceeds the latter in quality and distances the player from favourable results that reflect significant progress in learning.

The aforementioned reveals the need to redesign the actions of the didactic strategy and leads to intensify the work based on the multifactorial evaluation. The undesired outcomes in the process highlighted the importance of critical recognition by coaches of their responsibility as mediators in the process. Said analysis 
allowed to reorganize the preparation according to the quality of the methods and means that promote a developing teaching.

On the other hand, cohesion, interaction and communication were favoured, from the establishment of new group formations in the team. In addition to the increase in the training volume of the theoretical and psychological preparation. The number of confrontational parties rose as a function of setting new learning goals for the personal components of the process. After 18 more training sessions, a new assessment of learning in tactical activity was considered pertinent.

\section{Control experiment}

The experimental variant concludes with the verification of the final state reached in the evaluation of the learning of the tactical activity and the transformations in the modes of action of the pedagogical groups, from their preparation once the didactic strategy has been implemented. This stage involved observing training sessions and competitive matches, based on the planned control system.

On this evaluative occasion under match conditions, the individual tactical performance of the men's team players showed a significant evolution with a predominance of the Good category. Which was surpassed by the first-line players with a Very Good evaluation and an average effectiveness of $77.5 \%$, very close to the previous result. The progress in the chain of actions is remarkable, which raises the level of creation over the expected learning, which reaches $58 \%$ of effectiveness as a result of the reaction of the opposite.

For their part, second-line players moved to the Good category with an average effectiveness of $71 \%$, in the expected learning in both types of actions. Likewise, they exceed creation levels by $43 \%$ of effectiveness over successful actions. Such results allow identifying the significance of learning in the team's players.

Group tactical performance is valued as positive, despite the establishment of new connections in the team. All the possible variants are evidenced in order to favour interconnection in social interaction. From the offensive point of view, the tactical actions corresponding to the front line formations were evaluated as well with a $72 \%$ effectiveness. It is important to point out the stability in the cooperation between the group formations, which ensure the achievement of the actions. While, on the defensive, this aspect was evaluated as fair, with 64\% effectiveness. Deficiencies in doubling and support persist, essential indicators in defensive work.

Despite the results of the group work, the superior quality of learning is recognized, in a short period of time. In this sense, its extension is considered based on goals of greater scope. However, there are traits of a cooperative and developmental learning in both players and coaches when directing the process.

The evaluative approach to collective tactical learning in match conditions, involved observing the team in the preparation matches in the National Cup organized in March $2018.50 \%$ of the four matches in which it participated, selected starting from the competitive level of rivals.

In the first game, the collective tactical performance was observed from the defensive point of view. The 4-2 and 3-2-1 leading defensive systems were used. The level of relations was affected by the rapid transitions of the opponent, as they did not allow the withdrawal and the reestablishment of numerical equality. It was positive to observe the variations operated, based on the insufficient effectiveness of the advanced systems. In this sense, the simple 6-0 system was chosen. In this way, a better $70.14 \%$ effectiveness was achieved 
and the concretion of the collective tipping actions. Consequently, deterrence, opponent swaps, and brand dubbing were favoured.

During the second game, the evaluation of the offense was prioritized. On this occasion, positive results were observed compared to the previous game. Specifically, 51 actions out of 81 were completed for a $62.96 \%$ effectiveness. The counterattack actions for the solution of the situations expressed were consistent. The level of creation showed a $49.01 \%$ effectiveness and highlighted the game as a generator of learning by raising the percentages of effectiveness to the category of good.

On the basis of learning in the game itself, the spatial advantage was achieved, which is based on the numerical balance, after the transition from defence to offense. In this sense, the development of the counterattack is favoured. The conjugation of three of the pre-established actions in the PEA of the tactical activity based on the creation of spaces constitutes a significant piece of information, if the derivation of seven variants is added. In this way, a higher level of creation is revealed.

The multifactorial evaluation of learning from the final considerations of the control system, allows to infer the need for a totalizing vision of the PEA in the tactical activity in the selection of handball in Honduras. In this sense, the qualitative correlation between each of the tests up to the general result is stressed.

The applicability of the control system for the multifactorial evaluation of learning is verified both in training conditions and in matches. Their instruments highlight the reliability for the interpretation of the results. On the other hand, the evaluation process made it possible to appreciate superior quality indicators on each occasion. The commitment of the players and the team to their own learning and its concrete expression in competitive performance is remarkable. Consequently, the positive impact of the applied proposal in the fulfilment of the programmed objectives in the chosen timing is affirmed.

The development of the actions conceived in the PEA during the match was conditioned to the interaction of the opponent, which prevented their repetition on many occasions. The foregoing led to the conception of variants, which in turn constituted new actions and demanded the creation of other solutions. As a result, an intention to move towards creation emerges, a reflection of the team's level of independence as the protagonist of its own learning.

Specifically, the results reveal a good individual and group tactical performance of the players and the team in different situations, when designing and implementing their metacognitive strategies for the achievement of self-learning. It is remarkable the presence of signs of a meaningful and cooperative learning that leads to the developmental vision of the process. The results obtained in the evaluated period require a new diagnosis in order to redesign the process.

Based on the evaluative analysis, the pedagogical group expressed motivation and interest in the results of each phase of the pre-experiment. Active and leading actions prevailed during preparation and especially in evaluation. In the same way, they demonstrated modes of professional performance in correspondence with the demands of the process, based on the internal feedback of the system and the recognition of its didacticmethodological deficiencies. From the evaluations the didactic strategy is enriched. 


\section{DISCUSSION}

The results obtained in the pre-experiment with the application of the control system allow to show the transformations in the educational practice, they show the modifications made in the development of the PEA of the tactical activity in the handball team of Honduras. These results, due to their importance for the group of trainers, nourish them with knowledge in such a way that they understand the need to diversify and systematize scientific and methodological work based on the subject, accept and apply the multifactorial perspective of the evaluation of learning in the activity tactics in the handball team, increase their level of didactic-methodological preparation, which allows them to contextualize the process and encourages the integration of their personal and non-personal components, transformations in the modes of professional performance depending on the direction and conception of the PEA of the tactical activity, have a developmental, meaningful and collaborative vision of the multifactorial evaluation of the process under study in training or competition scenarios. In the team, it is relevant that they understand the manifestation of protagonism in the multifactorial evaluation as a process, they can assess from their initial state towards the development of the PEA they show superior tactical learning through their performance, with notable incidents in the collective work, also that this reveals the transit of an interactive developer controversy that contributes to the achievement of positive interdependence from the individual to the collective level and vice versa, promoting quality in the creation of variants, based on those established in the PEA of the tactical activity in the team handball, with a notable preference towards offense over defence; have a better enjoyment of the learning assessment tasks and recognition of their usefulness for better tactical performance in both training and competition scenarios.

\section{CONCLUSIONS}

The results obtained show the usefulness of the control system which allows evaluating the tactical activity in the context of learning in the sports training process, as well as its relevance and feasibility, being of great significance taking into account that it connotes a multifactorial evaluation of the tactical activity to promote a higher quality in the process under study.

It starts from recognizing the enrichment of the control system from the contributions and experiences of the practice in the training process. In this sense, the feedback of the subsystems is assumed based on the redesign of the actions. Therefore, it is important to attend to the incidence of the modifications or regulations in the essence to determine the effectiveness of the evaluation and its developer contribution.

\section{REFERENCES}

Acedo, A. (2017). Handball statistics.

Appz, W. T. (2017). Handball statistics. Retrieved from: https://play.google.com/store/apps/details?id=com.whattheappz.handball

Feu, S. (2006). Didactic organization of the teaching-learning process for the construction of the offensive game in handball.

Forcades, L. (2008). Handball: selection of texts. Havana, Editorial Sports.

García, J. (2008). The assessment of learning. Editorial Sports Havana.

García, J. (1998). Evaluation and control in sport. Spain. Gymnos Editorial.

González, I. (2015). Analysis of offensive and defensive behavior in handball through performance indicators and efficiency indices: validation of a software in real time. PhD thesis, University of Vigo. 
González, I. (2015). Technological tools for the analysis of the game in handball in real time. Readings: Physical education and sports, (202): 13.

LLC, M. (2017). Sportboard: Handball Board. Retrieved from: http://mokyn.com/sb/list.php?sportstype=HB

Lo Giudice, V. (2016). Assistant coach handball. Retrieved from: https://www.assistantcoach.co/

Martín, I., González, A., Cavalcanti, L. A., Chirosa, L. J., and Aguilar, J. (2013). Reliability and optimization of the protodeba v 1.0 program for observing handball decision making. Notebooks of Sports Psychology, 13 (1): 63-70. https://doi.org/10.4321/S1578-84232013000100007

Martínez, E., Olavarrieta, D.M., Milián, V (2018) System for the analysis of significant tactical actions of handball teams. Cuban Journal of Computer Science. 12 (1).

Martínez, R. and others. (2011). Observation protocol for the control of tactical behavior in basketball players aged 9 and 10 in the Florida municipality.

Mesa, L. (2007). Methodology for the control of the technical tactical activity in the handball goalkeeper. Thesis as an option to the scientific degree of Doctor in Physical Culture Sciences.

Ortega, A. J. (2009). Methodology for the study of offensive tactical thinking in the soccer team category 15-16 years of the Cucuta deportivo de norte de Santander Colombia. Degree thesis (Doctor of Physical Culture Sciences) Havana, ISCF "Manuel Fajardo".

Pensado, J. \& Russell González, L. (2012). Model for evaluating offensive tactical performance in sports games. Conference given at CETHLON - Center for Physical Education and Sports Studies UCCFD Manuel Fajardo, Cuba - First Scientific Event.

Quintana, D. (2014). Control of competitive performance in handball teams. Thesis as an option to the title of Doctor of Physical Culture Sciences. Matanzas School of Physical Culture.

Refoyo, I. (2001). The tactical decision of the game and its relationship with the biological response of the players: an application to basketball as a team sport. Complutense University Madrid.

Román, J. (2008). Collective group tactic in attack: the models in Spanish handball. Lo BM Seirul-lo, F. (2003). Dynamic systems and performance in team sports. 1st Meeting of Complex and Sport. INEFC-Barcelona.

Salas, J. J. and Mendo, A. H. (2016). Analysis of the data quality and generalizability of a counterattack observation system in elite handball. E-handball. com: Journal of Sports Sciences, 12 (1).

Sommerville, I. (2005). Software Engineering: A Practical Approach. Eddison Wesley, Mexico.

Villa, O. A., Rodríguez, J. H., and Rodríguez, C. R. S. (2016). Indicators for the control and evaluation of the technical-tactical preparation in handball. E-handball. com: Journal of Sports Sciences, 12 (1): 65-77.

Yucra, J. (2001). The evaluation in the sports training process.

\section{(@) $\odot \Theta \Theta$}

This work is licensed under a Attribution-NonCommercial-NoDerivatives 4.0 International (CC BY-NC-ND 4.0). 